\title{
Review of: "Oxygen/Ozone Therapy: a promising approach for the treatment of biphosphonate-related osteonecrosis of the jaw"
}

\author{
Božana Lončar-Brzak
}

Potential competing interests: The author(s) declared that no potential competing interests exist.

The study reports results about the treatment of MRONJ with oxygen/ozone therapy, updated after preliminary data published by Goker and al. (1) where these authors are also coauthors. Goker and al. have included 14 patients in their study while the authors of this study report results which include 16 patients, by the same protocol. The results are reported without using statistical methods or overall success rate after treatment, nor they are compared with other results (the paragraph Discussion is missing).

It seems that additional two patients do not make substantial difference in the results when compared with previously published data. Also, the photographs which are associated with this article show the same patient already shown in the paper of Goker and al.

This paper does not bring new and unpublished insights and is not for publication. 